\title{
Chronic kidney disease and cognitive impairment in the very old
}

\author{
Tomoyuki Kawada ${ }^{1}$
}

Received: 18 November 2015/Accepted: 23 December 2015/Published online: 28 January 2016

(C) Springer International Publishing Switzerland 2016

\section{To the Editor}

Wang et al. [1] determined whether chronic kidney disease (CKD) was associated with cognitive impairment among nonagenarians and centenarians in China. Among 767 subjects, there were $113(14.7 \%)$ and $577(75.2 \%)$ cases of $\mathrm{CKD}$ and cognitive impairment, respectively. The 30-item mini-mental state examination (MMSE) was used for the assessment of cognitive function, and estimated glomerular filtration rate (eGFR) $<60 \mathrm{~mL} / \mathrm{min} / 1.73 \mathrm{~m}^{2}$ by Chinese version of the Modification of Diet in Renal Disease (MDRD) equation was used to determine CKD. By logistic regression analysis, there was no association between cognitive impairment and CKD, although age, gender, education and income were significantly associated with cognitive impairment. I have two comments on their study.

First, the authors used MDRD equation for eGFR, which was determined by gender, age, and serum creatinine. The authors also used gender and age as independent variables for cognitive impairment, and odds ratios of these variables became significant by adjusted logistic regression analysis. I suppose that there is no problem of using gender and age for eGFR and each independent variable simultaneously. But caution should be paid on the unstable estimate of eGFR in the very old, who became emaciated. On this point, the authors used obesity indices as independent variables.
Tomoyuki Kawada

kawada@nms.ac.jp

1 Department of Hygiene and Public Health, Nippon Medical School, 1-1-5 Sendagi, Bunkyo-Ku, Tokyo 113-8602, Japan
Second, the authors used MMSE for the assessment of cognitive impairment, and about $3 / 4$ subjects were judged as subjects with cognitive impairment. Namely, majority of subjects were judged as having positive outcome. Life expectancy at birth in China in 2012 is 75.2 years, and the authors handled relatively healthy subjects. This situation presented the relatively small contribution of CKD or other physical disorders to cognitive impairment. In contrast, educational levels and independent incomes were found to contribute to prevention of cognitive impairment.

\section{Compliance with ethical standards}

Conflict of interest None.

Ethical approval This article does not contain any studies with human participants or animals performed by any of the authors.

Informed consent For this type of study a formal consent was not required.

\section{Reference}

1. Wang H, Fang C, Cai L et al (2015) Chronic kidney disease and cognitive impairment among the very old in China. Aging Clin Exp Res. doi:10.1007/s40520-015-0433-1 